\title{
Esofagite eosinofílica
}

Luiz João Abrahão Junior

Luiz João Abrahão

Ana Cristina de Azevedo Abrahão Oliveira

\section{INTRODUÇÃO}

A esofagite eosinofílica (EoE), primeiramente descrita em 1978 como uma doença rara, nos últimos anos tem sido cada vez mais diagnosticada em pacientes com disfagia, impactação alimentar e/ou pirose.

Há controvérsia quanto ao aumento na incidência da EoE, sendo atribuída em algumas séries ao maior conhecimento da doença por parte dos médicos (maior número de diagnósticos), embora alguns defendam que um aumento real do número de casos esteja ocorrendo ${ }^{1}$.

Trata-se de uma doença crônica, imunomediada, caracterizada por infiltração de eosinófilos na mucosa e submucosa esofágica.

Sua causa permanece desconhecida, sendo atribuída a fenômenos alérgicos (alérgenos alimentares ou inalados) e/ou imunológicos.

Critérios para o diagnóstico foram recentemente revistos e compreendem:

- Presença de sintomas de disfunção esofágica.

- Evidência histológica de eosinofilia esofágica (limitada apenas ao esôfago, e não em outros órgãos): no mínimo 15 eosinófilos intraepiteliais por campo de maior aumento.

- Remissão da eosinofilia com dietas de exclusão ou corticosteroides tópicos.

- A eosinofilia esofágica não deve responder ao tratamento com inibidores de bomba de prótons (IBP) isoladamente ${ }^{16}$. 


\section{EPIDEMIOLOGIA}

A EoE acomete mais frequentemente jovens entre 30 e 40 anos e do sexo masculino (relação masculino/feminino de 3:1), embora já tenha sido descrita em qualquer idade. Um estudo descreveu maior incidência entre caucasianos não hispânicos ${ }^{2}$, embora já tenha sido descrita em afro-americanos, asiáticos e latino-americanos.

Agrupamentos familiares também são descritos, embora não haja ainda estudos genéticos em adultos. Acredita-se que um dos genes responsáveis pelo agrupamento familiar seja a eotaxina-3.

\section{FISIOPATOLOGIA}

A fisiopatologia da EoE, embora ainda desconhecida, parece estar relacionada à alergia a alimentos ou aeroalérgenos.

A reação alérgica (a alimentos ou aeroalérgenos) parece ser mediada por IL-5 e eotaxina-3, que induzem a remodelação esofágica mediada por eosinófilos.

Aglomerados familiares de portadores de EoE sugerem a existência de predisposição genética ou exposição comum a alérgenos, embora a descoberta de maior expressão do gene que decodifica a eotanina- 3 em pacientes com EoE apóie a primeira hipótese $e^{3}$.

\section{QUADRO CLÍNICO}

Os sintomas de apresentação mais frequentes no adulto são disfagia, impactação alimentar, pirose e dor torácica ${ }^{4}$.

O sintoma mais comum é a disfagia para sólidos, ocorrendo em 60 a $90 \%$ dos casos, seguido de impactação alimentar em 50 a 60\% dos casos.

Sintomas de doença do refluxo gastroesofágico (DRGE) como pirose e regurgitação ácida são encontrados em 24 a $50 \%$ dos $\operatorname{casos}^{5,6} \mathrm{e}$ a elevada prevalência desses sintomas faz com que alguns pacientes sejam diagnosticados após serem considerados portadores de DRGE refratária ao tratamento.

Em crianças, os sintomas mais frequentes são dor abdominal, vômitos, regurgitação, baixo crescimento e dificuldade na alimentação.

Eosinofilia periférica é relatada em até $30 \%$ dos casos e geralmente é leve a moderada ${ }^{5}$.

É comum a associação entre EoE e outros processos alérgicos imunomediados em um mesmo paciente, destacando-se asma, rinite alérgica e alergias cutâneas e alimentares. 


\section{DIAGNÓSTICO ENDOSCÓPICO}

Aproximadamente 10 a 33\% dos pacientes portadores de EoE apresentam mucosa esofágica macroscopicamente normal à endoscopia digestiva, sendo diagnosticados apenas por meio do exame histopatológico ${ }^{4,5,7}$.

Os achados endoscópicos clássicos da esofagite eosinofílica compreendem a formação de anéis mucosos (traquealização ou felinização do esôfago), granulações brancas elevadas de mucosa que podem representar microabscessos de eosinófilos, sulcos longitudinais, exsudatos brancos e mucosa em "papel crepe" (mucosa friável que rasga à simples passagem do aparelho) (Figura 1).

Estenoses podem ocorrer em qualquer segmento do esôfago, isto é, esôfago proximal, médio ou distal.

A presença de um dos sinais descritos se correlacionou ao achado histopatológico de EoE em apenas 38\% dos pacientes, sendo a correlação maior quando duas ou mais alterações são identificadas no mesmo paciente ${ }^{6}$.

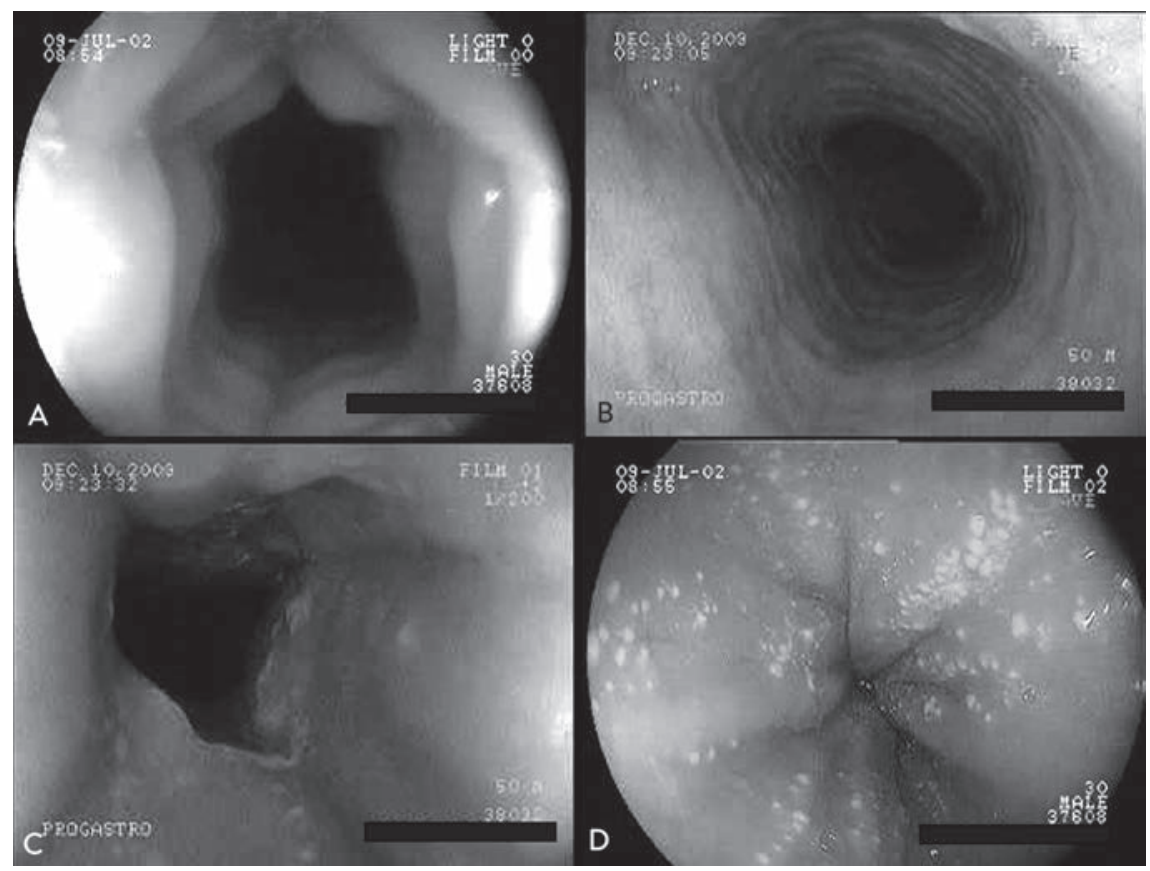

Figura 1 Achados endoscópicos na esofagite eosinofílica. A e B: Anéis mucosos (traquealização ou felinização do esôfago) e sulcos longitudinais. C: Estenose anelar em esôfago distal. D: Pontilhado branco em esôfago distal (microabscessos de eosinófilos). 
Para o diagnóstico histopatológico da EoE, múltiplas biópsias devem ser realizadas ao longo de toda a extensão do esôfago, mesmo em áreas macroscopicamente normais ${ }^{4}$. Recomenda-se que se obtenham ao menos quatro fragmentos de cada segmento do esôfago.

\section{DIAGNÓSTICO HISTOPATOLÓGICO}

A presença de infiltrado eosinofílico na mucosa esofágica faz o diagnóstico de EoE.

Vários critérios para o diagnóstico histopatológico foram propostos, dentre eles a contagem acima de 24 eosinófilos por campo de maior aumento e mais recentemente o proposto pelo consenso norte-americano de 15 eosinófilos por campo de maior aumento (4003) na área de infiltrado eosinofílico mais denso ${ }^{4}$ (Figura 2).

A EoE pode apresentar um padrão de acometimento heterogêneo, em placas, o que pode em alguns casos dificultar a confirmação da doença; quanto menor o número de fragmentos de biópsias obtidos, menor a sensibilidade diagnóstica.

Outros achados que podem ser observados em biópsias de pacientes com EoE são microabscessos de eosinófilos (aglomerados de mais de quatro eosinófilos), infiltrado eosinofílico, preferencialmente na metade superior da mucosa esofágica, hiperplasia da camada basal, alongamento das papilas e edema epitelial ${ }^{1}$.

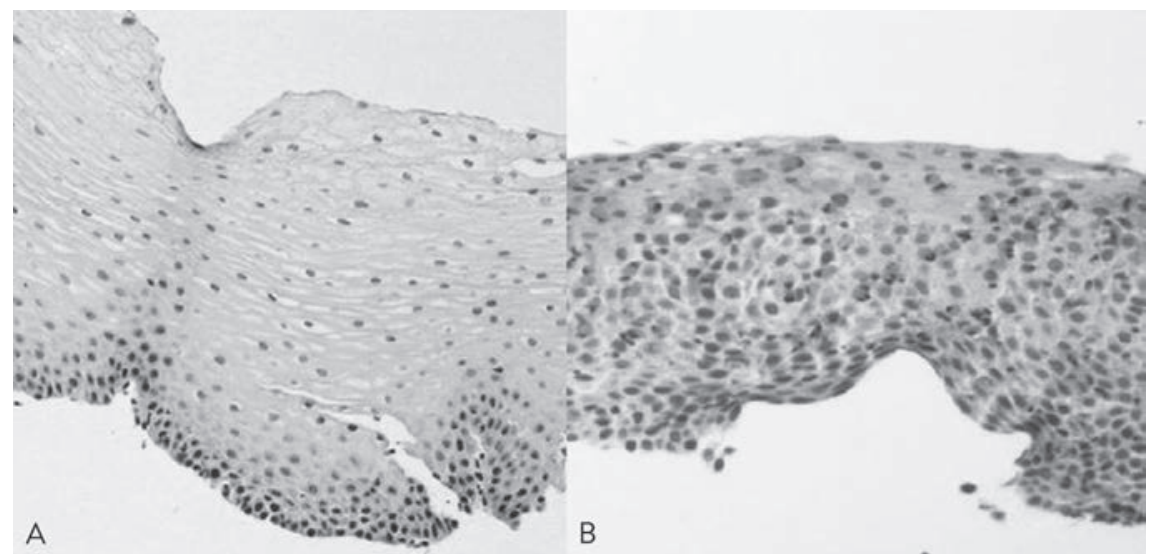

Figura 2 Achados histopatológicos da mucosa esofágica normal (A) e em paciente com esofagite eosinofilica (B) (HE magnificação de 2003). 
Estudos recentes têm demonstrado que 39 a 75\% dos pacientes com eosinofilia esofágica ( $>15$ eos/CMA) podem responder completamente ao tratamento com IBP isoladamente, sendo importante excluir a DRGE (ou com tratamento empírico ou por meio de pHmetria) antes de estabelecer o diagnóstico de $\mathrm{EoE}^{17}$.

Outras causas de eosinofilia devem ser descartadas, como gastroenterite eosinofílica, doença celíaca, síndrome hipereosinofílica, doença inflamatória intestinal e infecções (esquistossomose, toxocaríase, anisaquíase).

\section{ESOFAGOMANOMETRIA}

A esofagomanometria é normal na maioria dos pacientes com EoE. Em uma série de 77 pacientes com EoE, 30 (39\%) apresentavam esofagomanometria anormal, em sua maioria alterações inespecíficas, e apenas um paciente apresentava critérios para espasmo esofágico difuso ${ }^{8}$.

Pouco se sabe sobre a causa da disfagia em pacientes com EoE sem estenose. Acredita-se que o processo inflamatório e fibrose da mucosa e submucosa resultem na redução da complacência esofágica, o que provocaria o sintoma de disfagia. Um estudo recente também demonstrou, por meio da ultrassonografia (USG) intraluminal de alta frequência, disfunção isolada da musculatura longitudinal do esôfago com preservação da atividade da musculatura circular, o que justificaria a ocorrência de manometria normal na maioria dos pacientes com EoE'.

Outro estudo empregando o EndoFlip em 33 pacientes com EoE demonstrou menor distensibilidade e complacência do corpo esofágico quando comparados a 15 controles $^{18}$.

Utilizando a USG intraluminal de alta frequência associado à esofagomanometria, foram estudados quatro pacientes com EoE quanto às propriedades biomecânicas do esôfago e comparados com seis controles, tendo confirmado que na EoE a musculatura longitudinal apresenta redução significativa da sua atividade e embora a distensibilidade tenha sido menor na EoE, a diferença não atingiu significância estatística ${ }^{19}$.

A esofagomanometria também é utilizada para localização do limite superior do esfíncter inferior para realização da pHmetria prolongada.

PHMETRIA PROLONGADA

A pHmetria prolongada tem grande utilidade na exclusão de DRGE em pacientes em que o quadro clínico, a endoscopia e as biópsias são inconclusivas para EoE. 
Em pacientes com diagnóstico de EoE, a pHmetria prolongada pode revelar associação com DRGE em até $10 \%$ dos casos ${ }^{5}$.

\section{ESOFAGOGRAFIA}

A esofagografia tem grande valor nos pacientes portadores de estenoses complexas ou naqueles em que se suspeita clinicamente de estenose, para programação da endoscopia e de possível dilatação.

\section{TRATAMENTO}

O objetivo primário do tratamento da EoE é o alívio dos sintomas, principalmente da disfagia, e a prevenção de complicações, sendo a mais temida a estenose esofágica.

Alguns autores também adotam a redução no número de eosinófilos por campo de maior aumento como parâmetro de resposta terapêutica, embora não exista consenso quanto ao valor de corte ideal, sendo descritos menos de 5 a 15 eosinófilos por campo de maior aumento ${ }^{10}$.

Há quem recomende também na avaliação da resposta terapêutica a regressão de marcadores histológicos de inflamação, como hiperplasia de células basais e fibrose subepitelial.

As drogas frequentemente empregadas no tratamento da EoE são os corticosteroides, os IBP, os antagonistas de leucotrienos e os imunomoduladores, sendo também empregados tratamentos dietéticos e endoscópicos (dilatação endoscópica de estenoses) em casos selecionados.

$\mathrm{O}$ atual consenso recomenda como terapia inicial o uso de IBP por 12 semanas, com repetição da endoscopia com biópsias. Nos casos de resposta clínica e histopatológica aos IBP, dá-se o nome de eosinofilia esofágica responsiva aos IBP, subtipo frequente de EoE e presente em até 50 a $60 \%$ de algumas séries de casos.

\section{Corticosteroides}

Corticosteroides sistêmicos e locais são potentes drogas capazes de induzir a remissão em pacientes com EoE.

Em pacientes com formas leves a moderadas da doença, a preferência inicial é pelo tratamento tópico com a fluticasona (440 ou $500 \mu \mathrm{g}$, a cada $12 \mathrm{~h}$ ), budesonida ou beclometasona por 4 a 6 semanas. O paciente deve aplicar a medicação sem o espaçador e deglutir a medicação, sendo recomendado que interrompa a ingestão de líquidos e alimentos até 30 minutos após a aplicação. 
Casos graves ou refratários ao tratamento tópico requerem o uso de corticoterapia sistêmica nas doses de até 1 a $2 \mathrm{mg} / \mathrm{kg}$ de prednisolona. Seu uso deve ser limitado face aos efeitos colaterais a longo prazo, não devendo ser usado como terapia de manutenção ${ }^{1}$.

Recidiva sintomática e histológica ocorre 3 a 6 meses após a interrupção da medicação na maioria dos casos, não havendo consenso quanto ao tempo ideal de tratamento e estratégia de manutenção.

\section{Antagonistas de leucotrienos}

Montelucaste, um antagonista de leucotrienos utilizado no tratamento da asma e da rinite alérgica, foi usado no tratamento da EoE com resultados contraditórios.

Nas doses de 10 a $40 \mathrm{mg} /$ dia por 4 meses, foi capaz de induzir remissão clínica, mas não histológica em 7 de 8 pacientes com EoE ${ }^{11}$.

$\mathrm{O}$ atual consenso sobre EoE não recomenda o uso desse medicamento para o tratamento.

\section{Supressão ácida}

A DRGE pode causar eosinofilia esofágica (geralmente inferior a 5 eosinófilos por campo de maior aumento), embora pouco se saiba sobre a inter-relação entre DRGE e EoE. As duas podem apenas coexistir ou uma pode preceder e predispor a outra e vice-versa.

Dos pacientes com EoE, 10 a 18\% apresentam exposição ácida anormal à pHmetria, sendo descritos na literatura pacientes com quadro clínico, endoscópico e histológico de EoE que respondem bem à supressão ácida com IBP ${ }^{10}$.

\section{Imunomoduladores}

Mepolizumab, um anticorpo monoclonal humanizado anti-IL5, demonstrou resultados contraditórios em dois estudos abertos. Houve melhora sintomática após 4 a 12 semanas de tratamento sem que houvesse remissão histológica.

Omalizumab, um anticorpo monoclonal anti-IgE, foi utilizado em um estudo em nove adultos demonstrando melhora sintomática e na eosinofilia periférica e esofágica ${ }^{12}$.

Infliximab foi empregado em um estudo em três pacientes com EoE sem que houvesse remissão clínica nem histológica. 
Azatioprina e 6-mercaptopurina (6-MP) foram empregados em pacientes dependentes de corticosteroides sistêmicos, tendo permitido a interrupção da corticoterapia sem reativação da doença ${ }^{13}$.

\section{Dieta}

O tratamento dietético é comprovadamente eficaz em crianças, com taxas de sucesso entre 77 e $98 \%$. Três abordagens dietéticas podem ser utilizadas, sendo a primeira a utilização de dietas elementares ${ }^{14,15}$, a segunda empiricamente a não ingestão de alimentos potencialmente patogênicos como leite, ovos, trigo, soja, frutos do mar e amendoim, e finalmente a interrupção de alimentos indicados por testes alérgicos.

No caso da remoção empírica, a dieta restritiva deve ser mantida por 4 a 6 semanas e após atingir remissão clínica e histológica, os alimentos podem ser reintroduzidos um a um. Os alimentos mais frequentemente envolvidos na gênese da EoE são o leite e o trigo ${ }^{10}$.

\section{Doença refratária}

Define-se refratariedade ao tratamento quando ocorre persistência de sintomas e/ou da inflamação esofágica.

Pacientes em uso de corticosteroides tópicos devem ser questionados quanto à adesão e à forma de administração. A budesonida em gel possui eficácia comprovadamente superior à inalada. Aumento da dose do corticoide inalatório pode também ser uma estratégia útil em pacientes refratários. Casos mais graves podem se beneficiar com dietas elementares. Azatioprina ou 6-MP também podem ser utilizados em pacientes refratários ou dependentes da corticoterapia ${ }^{20}$.

Em pacientes com estenoses esofágicas que apresentam remissão da eosinofilia tecidual sem alívio da disfagia, dilatação endoscópica deve ser considerada.

Montelucaste, cromoglicato sódico, mepolizumab, reslizumab e omalizumab não foram eficazes em pacientes corticoide-dependentes ou refratários ${ }^{20}$.

\section{REFERÊNCIAS BIBLIOGRÁFICAS}

1. Prasad GA, Talley NJ. Eosinophilic esophagitis in adults. Gastroenterol Clin North Am. 2008;37(2):349-68.

2. Assaad AH, Putnam PE, Collins $\mathrm{MH}$, et al. Pediatric patients with eosinophilic esophagitis: an 8-year follow-up. J Allergy Clin Immunol. 2007;119(3):731-8.

3. Straumann A, Hruz P. What's new in the diagnosis and therapy of eosinophilic esophagitis? Curr Opin Gastroenterol. 2009;25(4):366-71. 
4. Furuta GT, Liacouras CA, Collins $\mathrm{MH}$, et al. Eosinophilic esophagitis in children and adults: a systematic review and consensus recommendations for diagnosis and treatment. Gastroenterology. 2007;133(4):1342-63.

5. Sgouros SN, Bergele C, Mantides A. Eosinophilic esophagitis in adults: a systematic review. Eur J Gastroenterol Hepatol. 2006;18(2):211-7.

6. Prasad GA, Talley NJ, Romero Y, et al. Prevalence and predictive factors of eosinophilic esophagitis in patients presenting with dysphagia: a prospective study. Am J Gastroenterol. 2007;102(12):2627-32.

7. Liacouras CA, Spergel JM, Ruchelli E, et al. Eosinophilic esophagitis: a 10-year experience in 381 children. Clin Gastroenterol Hepatol. 2005;3(12):1198-206.

8. Chen JGS, Pandolfino J, Kahrilas PJ, et al. Esophageal dysmotility in eosinophilic esophagitis: analysis using high resolution esophageal motility. Gastroenterology. 2007;132:SA6.

9. Korsapati H, Babaei A, Bhargava V, et al. Dysfunction of the longitudinal muscles of the oesophagus in eosinophilic oesophagitis. Gut. 2009;58(8):1056-62.

10. Gonsalves N, Kahrilas PJ. Eosinophilic oesophagitis in adults. Neurogastroenterol Motil. 2009;21(10):1017-26.

11. Attwood SE, Lewis CJ, Bronder CS, et al. Eosinophilic oesophagitis: a novel treatment using Montelukast. Gut. 2003;52(2):181-5.

12. Foroughi S, Foster B, Kim N, et al. Anti-IgE treatment of eosinophil-associated gastrointestinal disorders. J Allergy Clin Immunol. 2007;120(3):594-601.

13. Netzer P, Gschossmann JM, Straumann A, et al. Corticosteroid-dependent eosinophilic oesophagitis: azathioprine and 6-mercaptopurine can induce and maintain long-term remission. Eur J Gastroenterol Hepatol. 2007;19(10):865-9.

14. Markowitz JE, Spergel JM, Ruchelli E, et al. Elemental diet is an effective treatment for eosinophilic esophagitis in children and adolescents. Am J Gastroenterol. 2003;98(4):777-82.

15. Kelly KJ, Lazenby AJ, Rowe PC, et al. Eosinophilic esophagitis attributed to gastroesophageal reflux: improvement with an amino acid-based formula. Gastroenterology. 1995;109(5):1503-12.

16. Liacouras CA, Furuta GT, Hirano I, et al. Eosinophilic esophagitis: updated consensus recommendations for children and adults. J Allergy Clin Immunol. 2011;128(1):3-20.

17. Lieberman JA, Chehade M. Eosinophilic esophagitis: diagnosis and management. Immunol Allergy Clin North Am. 2012;32(1):67-81.

18. Kwiatek MA, Hirano I, Kahrilas P, et al. Mechanical properties of the esophagus in eosinophilic esophagitis. Gastroenterology. 2011;140:82-90.

19. Abrahao JR LJ, Lemme EMO, Henriques LO, et al. Propriedades biomecânicas do esôfago de pacientes com esofagite eosinofílica estudadas através da ultrassonografia intraluminal de alta frequência. Gastroenterologia Endoscopia Digestiva (GED). 2011;30(supl.4):243.

20. Sodikoff J, Hirano I. Therapeutic strategies in eosinophilic esophagitis: induction, maintenance and refractory disease. Best Pract Res Clin Gastroenterol. 2015; 29(5):829-39. 